\title{
A Role for the Locus Ceruleus in Reward Processing: Encoding Behavioral Energy Required for Goal-Directed Actions
}

\author{
T- Jeremy Hofmeister ${ }^{1,2,3}$ and Virginie Sterpenich ${ }^{1,2,4}$ \\ ${ }^{1}$ Laboratory for Neurology and Imaging of Cognition, Department of Neuroscience, Faculty of Medicine, ${ }^{2}$ Geneva Neuroscience Center, ${ }^{3}$ Medical Image \\ Processing Laboratory, Department of Radiology and Medical Informatics, Faculty of Medicine, and ${ }^{4}$ Swiss Center for Affective Sciences, University of \\ Geneva, 1211 Geneva 4, Switzerland
}

Review of Bouret and Richmond

The locus ceruleus (LC) is a small nucleus in the dorsal pons that innervates most of the forebrain, with the exception of the striatum. The LC is the main source of norepinephrine (NE) in the brain, and this LC-NE system is typically associated with arousal, vigilance states, attention, and emotions (Sara and Bouret, 2012). LC neurons exhibit both phasic and tonic firing modes: long-lasting changes in tonic signals (seconds or more) are thought to contribute to the transition between behavioral states (e.g., sleep, attention, exploration, or stress), while several hypotheses about the role of transient phasic activation (few hundreds of milliseconds) have been formulated. First, Aston-Jones and Cohen (2005) proposed that phasic LC activation provides a mechanism for optimizing behavioral performance by enhancing activity in a complex neural matrix involving sensory, motor, and decisional regions. Second, phasic LC responses have been suggested as a "network reset" signal, enhancing adaptive behavioral response by facilitating reorientation of corti-

Received April 20, 2015; revised June 16, 2015; accepted June 17, 2015. We thank Prof. Sophie Schwartz for insightful comments on the manuscript.

Correspondence should be addressed to Jeremy Hofmeister, Department of Neuroscience, Faculty of Medicine, University of Geneva, rue Michel-Servet 1, 1211 Geneva 4, Switzerland. E-mail: Jeremy.hofmeister@unige.ch.

DOI:10.1523/JNEUROSCI.1734-15.2015

Copyright $\odot 2015$ the authors $\quad 0270-6474 / 15 / 3510387-03 \$ 15.00 / 0$ cal networks toward the processing of salient stimuli (Sara and Bouret, 2012). Third, another closely related theory proposed that the LC-NE system influences the functional shift of activity between ventral and dorsal frontoparietal attentional networks, which are involved in stimulusdriven attention (i.e., the detection of salient stimuli in the environment) and goaldriven attention (i.e., the selection of stimuli based on personal goals and expectations), respectively (Corbetta et al., 2008). This shift of activity between goal-driven and stimulus-driven attention would ultimately enable the detection of selected salient sensory stimuli based on internal expectations and facilitate adaptive motor responses. Despite difference in the specifics of these hypotheses, most authors agree that phasic activation of the LC-NE system plays an important role in modulating goal-directed attentional processing and is tightly linked with behavioral performance.

Several recent studies demonstrate that the LC-NE system is also linked to motivation. For example, Bouret et al. (2012) report that LC responses are modulated by cues predicting reward size. Note that this modulation parallels similar modulation of dopaminergic (DA) neurons, which are crucially involved in reward prediction (Schultz, 2007; Glimcher, 2011). However, the unique contribution of LC-NE system to reward processing have not yet been elucidated. Hence, a recent study by Bouret and Richmond (2015) sought to investigate how LC activity relates to motivation, using single-neuron recording in monkeys. The authors propose that the LC plays a key role in directing the behavioral energy needed to perform goal-directed action.

The authors recorded the response of LC neurons of two monkeys while manipulating the size of rewards given during a task involving three types of trials: cuedactive, cued-passive, and self-initiated trials. Cued-active trials started with a cue indicating reward size when monkeys touched a bar. The cue was followed by a wait signal, during which monkeys had to keep holding the bar. After a variable delay, a go signal instructed the monkeys to release the bar rapidly. If they released the bar rapidly enough, a feedback display was shown, followed by reward delivery (according to the reward size indicated by the initial cue: 1, 2, or 4 drops of water). Cued-passive trials also started with a cue indicating reward size when monkeys touched a bar. However, once the cue appeared, releasing or touching the bar again had no effect. After a delay, a feedback signal was shown, followed by reward delivery, as for cued-active trials. Finally, in self-initiated trials, no cues were shown and monkeys had to hold and release the bar in a self-initiated manner. 
After releasing the bar, a feedback was shown, followed by reward delivery.

Bouret and Richmond (2015) found that monkeys were faster and more accurate as reward size increased in cuedactive trials (i.e., for trials requiring a goaldirected action following specific go signal). This observation replicates the known effect of reward size on operant performance (Bouret and Richmond, 2010).

To characterize LC activity during cue presentation (in cued-active and cuedpassive trials), around action initiation (i.e., shortly before feedback display in cued-active and self-initiated trials), and when processing feedback (i.e., right after feedback display in all trial types), the authors used an epoch-of-interest analysis. They found a general phasic activation of all LC neurons for all three periods. At cue onset, both in cued-active and cuedpassive trials, they reported a strong positive influence of reward size on LC activity (i.e., the larger the reward, the higher the LC activity). Interestingly, at action initiation in cued-active trials, LC activity was negatively modulated by reward size (i.e., the smaller the reward, the higher the LC activity). For the same period in selfinitiated trials (for which no cues were presented and that consequently were not previously positively influenced by expected reward size), LC neurons showed a strong activation, but no modulation by reward size. Feedback presentation elicited a small activation in cued-passive trials without modulation by reward size, and no additional activation in cuedactive or self-initiated trials, as they were both activated by action initiation shortly before.

Based on their results, Bouret and Richmond (2015) hypothesize that the activity of LC neurons reflects the behavioral energy required to perform goaldirected actions. The authors propose that when the initial cue is presented, LC neurons respond to mobilize energy for further operant action; because the monkeys want to mobilize more energy for larger rewards, LC activity is higher in these cases. If the initial amount of energy mobilized is insufficient to complete the task, then at the time of action initiation, the monkeys need to mobilize additional energy for optimal performance. Because this amount is inversely related to the amount of energy already mobilized at cue onset, LC neurons show greater activity for smaller rewards when initiating goal-directed action. As the task progressed, the authors found that this effect
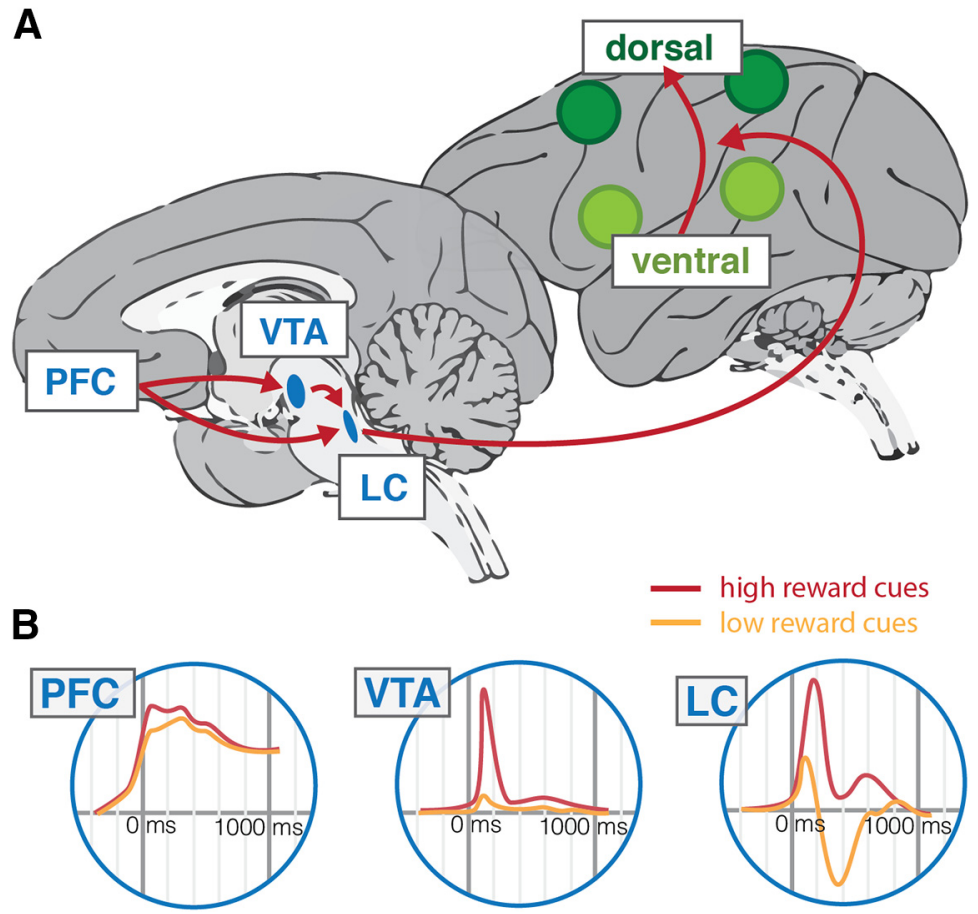

Figure 1. A proposed circuit for the processing of reward cues. $\boldsymbol{A}$, The prefrontal cortex (PFC) transmits reward size information to the VTA and target-detection signal to the LC. Then, the VTA conveys reward-prediction signal to the LC, which codes for behavioral energy required to perform goal-directed action based on expected reward. The LC ultimately facilitates the shift between ventral (light green) and dorsal (dark green) frontoparietal attentional network to increase behavioral performance. $\boldsymbol{B}$, Event-related activity in the PFC, VTA, and LC, for high (red line) and low (orange line) reward size. Note that time courses of the PFC, VTA, and LC are adapted from Bouret and Richmond (2010), Schultz (2007), and Bouret and Richmond (2015), respectively, and that $y$-axes have arbitrary scales.

became prominent, possibly because of a gradual increase in satiation, paralleled by a reduction in motivation.

The lack of modulation by reward size at the time of feedback is consistent with the authors' proposal that the LC-NE system does not represent reward directly but assumes a role in mobilizing energy or resources necessary to perform actions. But what does "mobilizing energy" mean? One possibility is that the LC-NE system activates hypothalamic networks that regulate energy expenditure (Saper and Lowell, 2014). Alternatively, we hypothesize that phasic LC firing during the processing of reward cues might influence the balance between ventral and dorsal frontoparietal attentional networks, relating to the above-mentioned hypothesis by Corbetta et al. (2008). According to such a model, when processing highly rewarding cues, the LC-NE system facilitates target detection (i.e., the go signal) by favoring transition to the dorsal attentional network (i.e., goal-directed attention). When processing less rewarding cues, the LC-NE system is less activated, and goal-directed attention is less favored. Later, when action is initiated to obtain a small expected reward, the LC-NE system has to compensate for the weak recruitment of the dorsal attentional network to adequately perform the action. However, a sufficient early transition to the dorsal attentional network would result in better behavioral performance, which is precisely what the authors observed for highly rewarded trials.

Bouret and Richmond (2015) also characterized the onset and peak latencies of event-related changes in firing rate for each type of trial and reward size. They found that the firing rate of LC neurons showed a biphasic increase after cue presentation: LC activity initially increased rapidly and uniformly for all cues, before showing differences across reward sizes (ultimately peaking $\sim 200 \mathrm{~ms}$ after cue presentation; Bouret and Richmond, 2015, their Table 1). Interestingly, the modulation of LC by reward size appeared later than what has been observed in the orbitofrontal cortex $(\sim 60 \mathrm{~ms}$; Bouret and Richmond, 2010) and in the ventral tegmental area (VTA, $\sim 100 \mathrm{~ms}$; Schultz, 2007 ), the origin of most DA activity involved in reward prediction.

Phasic LC responses to stimuli are thought to occur relatively late, after the decision signal, and to be triggered by prefrontal (PFC) inputs that focus LC activity 
on target stimuli (Aston-Jones and Cohen, 2005). In the task used by Bouret and Richmond (2015), all cues are target stimuli and monkeys learn to perform well for all cue types. However, trials associated with higher rewards are more appetitive, and monkeys are willing to invest more resources to perform these trials successfully. The biphasic response of the LC to cues might reflect these two aspects when processing reward-predicting cues: (1) target-stimulus detection (independent of reward size), and (2) recruitment of behavioral resources according to predicted reward size. Hypothetically, the initial increase in LC activity for all cues may be triggered by PFC inputs and reflect detection of target stimuli, as reported by Rajkowski et al. (2004). The later modulation of LC by reward size might be triggered by the VTA after it computed the predicted reward value. Indeed, the connections between VTA and LC are rapid and reciprocal (Chandler et al., 2014), and the reward-related signal appears earlier in the VTA (at $\sim 100 \mathrm{~ms}$ ) than in the LC (at $\sim 200 \mathrm{~ms}$ ). Thus, when processing cues predicting high reward, the VTA might activate the LC to improve behavioral performance.

In summary, Bouret and Richmond (2015) proposed that the role of the LC is related to the behavioral energy required to perform goal-directed actions. Based on their findings and recent literature, we hypothesize that phasic LC reward modulation when processing highly cues might be triggered by VTA, and that the recruit- ment of the LC might be used to reorient attentional networks toward the detection of salient stimuli to increase behavioral performance (Fig. 1). Future research should examine whether LC activity also varies during tasks involving the avoidance of different levels of negative outcomes (e.g., losses or punishments) to better define the general framework of phasic LC activation when initiating goaldirected actions. Additionally, it may be valuable to investigate whether changes in tonic LC firing modulate the processing of rewards and losses, as studies reported here mostly investigated phasic LC firing. Indeed, studies in humans found that people with lower levels of the norepinephrine transporter (and therefore more NE in the brain) have higher loss aversion (Takahashi et al., 2013), and emphasized the role of arousal in aversion to losses (Sokol-Hessner et al., 2009).

\section{References}

Aston-Jones G, Cohen JD (2005) An integrative theory of locus coeruleus-norepinephrine function: adaptive gain and optimal performance. Ann Rev Neurosci 28:403-450. CrossRef Medline

Bouret S, Richmond BJ (2010) Ventromedial and orbital prefrontal neurons differentially encode internally and externally driven motivational values in monkeys. J Neurosci 30: 8591-8601. CrossRef Medline

Bouret S, Richmond BJ (2015) Sensitivity of locus ceruleus neurons to reward value for goaldirected actions. J Neurosci 35:4005-4014. CrossRef Medline

Bouret S, Ravel S, Richmond BJ (2012) Complementary neural correlates of motivation in dopaminergic and noradrenergic neurons of monkeys. Front Behav Neurosci 6:40. CrossRef Medline

Chandler DJ, Waterhouse BD, Gao WJ (2014) New perspectives on catecholaminergic regulation of executive circuits: evidence for independent modulation of prefrontal functions by midbrain dopaminergic and noradrenergic neurons. Front Neural Circuits 8:53. CrossRef Medline

Corbetta M, Patel G, Shulman GL (2008) The reorienting system of the human brain: from environment to theory of mind. Neuron 58 : 306-324. CrossRef Medline

Glimcher PW (2011) Understanding dopamine and reinforcement learning: the dopamine reward prediction error hypothesis. Proc Nat Acad Sci U S A 108[Suppl 3]:15647-15654. CrossRef Medline

Rajkowski J, Majczynski H, Clayton E, AstonJones G (2004) Activation of monkey locus coeruleus neurons varies with difficulty and performance in a target detection task. J Neurophysiol 92:361-371. CrossRef Medline

Saper CB, Lowell BB (2014) The hypothalamus. Curr Biol 24:R1111-R1116. CrossRef Medline

Sara SJ, Bouret S (2012) Orienting and reorienting: the locus coeruleus mediates cognition through arousal. Neuron 76:130-141. CrossRef Medline

Schultz W (2007) Multiple dopamine functions at different time courses. Ann Rev Neurosci 30:259-288. CrossRef Medline

Sokol-Hessner P, Hsu M, Curley NG, Delgado MR, Camerer CF, Phelps EA (2009) Thinking like a trader selectively reduces individuals' loss aversion. Proc Natl Acad Sci U S A 106:5035-5040. CrossRef Medline

Takahashi H, Fujie S, Camerer C, Arakawa R, Takano H, Kodaka F, Matsui H, Ideno T, Okubo S, Takemura K, Yamada M, Eguchi Y, Murai T, Okubo Y, Kato M, Ito H, Suhara T (2013) Norepinephrine in the brain is associated with aversion to financial loss. Mol Psychiatry 18: 3-4. CrossRef Medline 